\title{
A Heuristic for the 3-staged 2D Cutting Stock Problem with Usable Leftover
}

\author{
Q.L. Chen \\ School of Business Administration \\ South China University of Technology \\ Guangzhou, China \\ College of Computer and Electronic Information \\ Guangxi University \\ Nanning, China \\ Y.D. Cui \\ College of Computer and Electronic Information \\ Guangxi University \\ Nanning, China
}

\author{
L.P. Li \\ College of Computer and Electronic Information \\ Guangxi University \\ Nanning, China \\ Y. Chen \\ School of Business Administration, \\ South China University of Technology, \\ Guangzhou, China \\ College of Computer and Electronic Information \\ Guangxi University \\ Nanning, China
}

\author{
X.Y. Lu \\ College of Computer and Electronic Information \\ Guangxi University \\ Nanning, China
}

\begin{abstract}
A heuristic approach is proposed for the 3-staged two-dimensional cutting stock problem with usable leftover, where the unused rectangular segments can be returned to stock for future use. The approach is based on the recursive technique combined with beam search. A forward recursion is used to generate normal segments that consist of homogenous strips, and a beam search heuristic is used to obtain the 3-staged cutting patterns considering usable leftover. The computational results show that the algorithm is effective in reducing material cost.
\end{abstract}

Keywords-3-staged cutting; beam search; recursive technique; usable leftover

\section{INTRODUCTION}

The two-dimensional (2D) cutting stock problem consists of seeking an optimal cutting plan that consists of different cutting patterns. We are particularly concerned with the special case where the non-used material (leftover) of the cutting pattern can be stocked for use in the subsequent cutting process, if large enough. With the usability of leftovers, a considerable amount of material can be saved.

The cutting stock problem with usable leftovers (CSPUL) was first introduced in the early 1970s by Brown [1]. Authors made extensive studies in the 1D CSPUL in the past two decades [2]. For the 2D CSPUL, only Andrade et al. [3, 4] deal with non-exact 2-staged (2NE) patterns. To the best of our knowledge, no paper deals with the 3-staged cutting pattern with usable leftovers.

This paper considers the 2D CSPUL with 3-staged patterns, where the unused rectangular segments of the cutting patterns can be stored as the usable leftovers. Each pattern contains only homogenous strips and is referred to as the homogenous 3-staged cutting pattern with usable leftover (3HL pattern).

\section{II.PROBLEM DESCRIPTION}

The paper proposes a heuristic algorithm to determine the cutting plan that consists of 3HL patterns, where a set of $m$ types of rectangular items are cut from stock plates of dimensions $L \otimes W$, each item type $i \in I=\{1, \cdots, m\}$, has a length $l_{i}$, a width $w_{i}$, a demand $d_{i}$ and a value $v_{i}$. The objective is to minimize the difference between the cost of the plate used and the value of usable leftovers.

If not specified, we assume that the cuts on the plate are infinitely thin, i.e. the saw thickness is zero. Otherwise the saw thickness is added to the dimensions of the plate and items.

\section{A. Usable Leftover}

A 3-staged pattern contains three stages of orthogonal guillotine cuts. If the first-stage cuts are vertical, it is an X-pattern, otherwise a Y-pattern. Only the unused segment along the pattern direction is considered as leftover. In other words, only the unused rectangular segment on the right of an $\mathrm{X}$-pattern or on the top of a Y-pattern can be considered as the potentially usable leftover (see Figure 1). As for that, the usable leftover has the same width, which is useful to simplify the cutting process and the inventory management. 


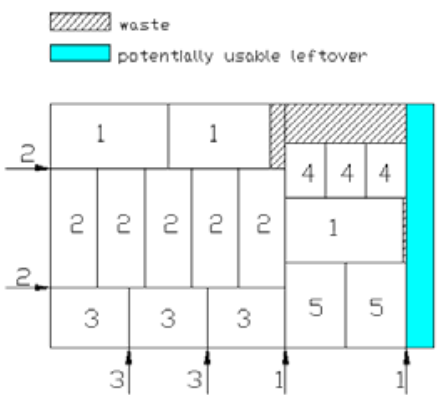

(a) X-pattern

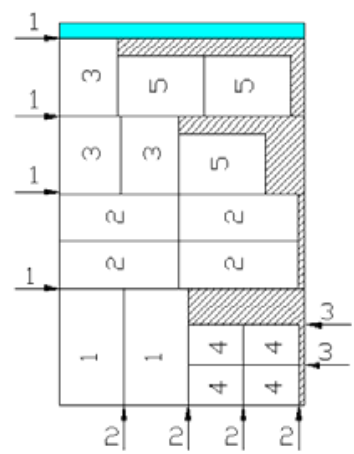

(b) Y-pattern
FIGURE I. 3-STAGED PATTERNS WITH USABLE LEFTOVER. (A) X-PATTERN. (B) Y-PATTERN.

\section{B. The Model for 3HL Pattern}

To simplify the description without loss of generality, the approach is described for the X-pattern with oriented items. It can be easily adapted for the Y-pattern and non-oriented items.

Let $l$ be the length of the usable leftover, $c$ be the unit value (value of unit area), $l_{\min }=\min _{i \in I}\left\{l_{i}, w_{i}\right\}$. The value $V_{l}$ of the leftover is defined as:

$$
V_{l}=\left\{\begin{array}{c}
c l W, \quad \text { if } l \geq l_{\text {min }} \\
0, \text { otherwise }
\end{array}\right.
$$

Let $z$ be the value of the 3HL pattern, $a_{i}$ be the number of type- $\boldsymbol{i}$ items included, $\mathbf{N}$ be the set of non-negative numbers. The following model determines the optimal 3HL pattern, where the objective is to maximize the pattern value (sum of the values of items and leftovers).

$$
z=\max \sum_{i=1}^{m} a_{i} v_{i}+V_{l} ; \quad a_{i} \leq d_{i}, a_{i} \in N, i \in I
$$

\section{ALGORITHM}

Both the X-pattern and Y-pattern are generated, and the better one is chosen as the optimal pattern. Assume that $V^{*}$, $V^{X}$ and $V^{Y}$ are respectively the optimal pattern value, the optimal $X$-pattern value, and the optimal Y-pattern value, then

$$
V^{*}=\max \left\{V^{X}, V^{Y}\right\}
$$

Items in the 3 -staged pattern are cut in three stages, subsequently the problem of finding the optimal 3HL $\mathrm{X}$-pattern can be divided into three procedures: strip-procedure, segment-procedure and pattern-procedure.

\section{A. Strip-Procedure}

A batch of strips $x \times w_{i}, i \in I_{x}=\left\{i \mid l_{i} \leq x\right\}$ are generated for segment $x \times W$. Initially the number of type- $i$ items in strip $x \times w_{i}$ is $e_{i}=\min \left(\left\lfloor x / l_{i}\right\rfloor, d_{i}\right)$, and the strip value is $v(x, i)=e_{i} v_{i}$.

\section{B. Segment-Procedure}

Normal segments $q_{k} \times W, q_{k} \in Q$ are used to improve the algorithm, where $Q$ is the set of normal lengths [5] .
Furthermore, this paper considers the adjacent segments jointly to get all the normal segments within a short time.

Forward recursion of dynamic programming is used to obtain the segment $x \times W$ through building strips $x \times w_{i}$, $i \in I_{x}$ on the top of sub-segment $x \times y$, to obtain a new sub-segment $x \times\left(y+w_{i}\right)$. The number of items in the sub-segment should not exceed demand, So $e_{i}=\min \left[\left\lfloor x / l_{i}\right\rfloor, d_{i}-n(x, y, i)\right]$, where $n(x, y, i)$ is the number of type- $i$ items included in the sub-segment $x \times y$. Update $v(x, i)$, and the value of the sub-segment $x \times\left(y+w_{i}\right)$ is:

$$
\begin{gathered}
\mathrm{F}\left(x, y+\mathrm{w}_{\mathrm{i}}\right)=\max \left\{\mathrm{F}\left(x, y+\mathrm{w}_{\mathrm{i}}\right), \mathrm{F}(x, y)+v(x, i)\right\}, \\
y \leq W-w_{i}, i \in I_{x}
\end{gathered}
$$

Where $F(x, y)$ is the value of sub-segment $x \times y$. Initially $F(x, 0)=0$.

Suppose that the solution of normal segment $q_{k} \times W$ is known as $\mathrm{F}\left(\mathrm{q}_{\mathrm{k}}, y\right)$. The length of its adjacent segment is $q_{k+1}$. If $q_{k} / l_{i}=q_{k+1} / l_{i}$, ignore item type $i$; Otherwise, add it to the new item set $I_{N}$. The initial value of the adjacent segment is $\mathrm{F}\left(\mathrm{q}_{\mathrm{k}+1}, y\right)=\mathrm{F}\left(\mathrm{q}_{\mathrm{k}}, y\right)$. Replace $I_{x}$ with $I_{N}$, and obtain the adjacent segment. The computation time of adjacent segments is reduced, because $\left|I_{N}\right|<<\left|I_{x}\right|$.

\section{Pattern-Procedure}

Beam search (BS) is used to generate 3HL patterns. BS is incomplete derivative of branch and bound algorithm where only elite nodes that have high potential are investigated. It is used to solve combinatorial optimization problems including two-staged cutting problems [6-7] and circular packing problem [8].

Three basic elements are required for a BS heuristic: node definition, branching strategy and evaluation operator. The node is defined by a pair of sub-rectangles $((x \times W),((L-x) \times W))$. Where $(x \times W)$ is the partial pattern that contains a set of normal segments with sum length equal to $X .((L-x) \times W)$ is the blank part remains to be filled. The root node is $((0 \times W),(L \times W))$, where no segment has been packed.

Branching on a node $\mu=((x \times W),((L-x) \times W))$ is equivalent to packing normal segments into the blank part, which creates a set of child nodes $\left(\left(\left(x+q_{k}\right) \times W\right)\right.$, $\left.\left(\left(L-x-q_{k}\right) \times W\right)\right),\left(L-x-q_{k}\right) \geq 0, \quad q_{k} \in Q$. Only the top $\delta$ potential child nodes are kept for further branching. The potential of a node $\mu$ is $\bar{z}_{\mu}=V_{P}+U_{(L-x)}$, where $V_{P}$ is the partial pattern value known as the sum value of the normal segments included. $U_{(L-x)}$ is the upper bound of $(L-x) \times W$.

$U_{x}$ can be obtained by solving the following knapsack problem: 


$$
U_{x}=\max \left\{\sum_{k \in K} F\left(q_{k}, W\right) t_{k} \mid \sum_{k \in K} q_{k} t_{k} \leq x, t_{k} \in N, k \in K\right\}
$$

Where $t_{k}$ is the frequency of segment $\left(q_{k} \times W\right)$, and $K=\left\{k \mid q_{k} \leq x, q_{k} \in Q\right\}$. When $U_{L}$ is obtained by solving the problem using the dynamic programming, all $U_{x}$ corresponding to sub-rectangles $x \times W(x=0,1, \cdots, L)$ are also obtained.

\section{Generation of Cutting Plan}

The Generation of the cutting plan contains three phases: (1) Generate the current 3HL pattern using the pattern-procedure, (2) update the remaining demands of the item types by deleting the items included in the current pattern; (3) Repeat Phases (1) and (2) until all the remaining demands become zero.

\section{COMPUTATIONAL RESULTS}

No algorithm for the 2D CSPUL with 3-staged patterns exists in the literature. So the computational results of $3 \mathrm{HL}$ patterns are compared with that of 2NE patterns with leftovers (2NEL patterns) and general guillotine patterns with leftovers (GGL patterns). Both the 2NE pattern and $3 \mathrm{H}$ pattern belong to GG pattern. The algorithm was coded in $\mathrm{C \#}$ and the computer used has clock rate $2.53 \mathrm{GHz}$ and main memory 2GB RAM. Set $\delta=2$, and $c=\frac{m}{|Q|} \cdot \min \left\{\frac{v_{i}}{L w_{i}}\right\}$.

\section{A. Compares with 2NEL Patterns}

The computational results of 3HL patterns in 12 instances are compared with the 2NEL patterns from Andrade [4]. Items are fixed orientation. The 3HL patterns use the same number of plates as the 2NEL patterns in all instances. Table 1 lists the computational results, where $P C$ is the plate cost, $L A_{3 H L}$ is the usable leftover area of 3HL patterns; $L A_{2 N E L}$ is that of $2 \mathrm{NEL}$ patterns, which contain two usable leftovers. The $3 \mathrm{HL}$ patterns get better solutions in 6 instances, equal in 5 instances and a little worse in 1 instance. The average computation time of 3HL patterns is 0.136 seconds. The average computation time for 2NEL patterns is unknown, for that the time (124.52s) includes the selection of plates.

TABLE I. COMPUTATIONAL RESULTS OF 12 INSTANCES.

\begin{tabular}{|c|c|c|c|c|c|c|c|}
\hline $\begin{array}{c}\mathrm{I} \\
\mathrm{D}\end{array}$ & $\begin{array}{c}\mathrm{P} \times \mathrm{W} \times N \\
)\end{array}$ & $\begin{array}{c}L A_{3} \\
H L\end{array}$ & $\begin{array}{c}L A_{2 N} \\
E L\end{array}$ & $\begin{array}{c}\mathrm{I} \\
\mathrm{D}\end{array}$ & $\begin{array}{c}L \times W \times N \\
)\end{array}$ & $\begin{array}{c}L A_{3} \\
H L\end{array}$ & $\begin{array}{c}L A_{2 N} \\
E L\end{array}$ \\
\hline 1 & $53 \times 52 \times 2$ & 530 & 520 & 7 & $19 \times 10 \times 2$ & 70 & 0 \\
\hline 2 & $63 \times 60 \times 2$ & $\begin{array}{c}306 \\
0\end{array}$ & 2898 & 8 & $132 \times 194 \times 2$ & $\begin{array}{c}\mathbf{1 6 2} \\
36\end{array}$ & $\begin{array}{c}1299 \\
8\end{array}$ \\
\hline 3 & $13 \times 10 \times 2$ & 52 & 52 & 9 & $14 \times 19 \times 1$ & 154 & 154 \\
\hline 4 & $18 \times 10 \times 2$ & 0 & 0 & $\begin{array}{c}1 \\
0\end{array}$ & $18 \times 10 \times 1$ & 18 & 18 \\
\hline 5 & $18 \times 10 \times 1$ & 108 & 108 & $\begin{array}{c}1 \\
1\end{array}$ & $14 \times 19 \times 1$ & $\mathbf{1 7 1}$ & 168 \\
\hline 6 & $16 \times 27 \times 2$ & 48 & 64 & $\begin{array}{c}1 \\
2\end{array}$ & $22 \times 17 \times 2$ & $\mathbf{1 3 2}$ & 0 \\
\hline
\end{tabular}

\section{B. Compares with GGL Patterns}

Two instances are used in this subsection, the saw thickness of Instance 1 is zero, and that of Instance 2 is 2.7 types of non-oriented items are cut from stock plate of dimensions $510 \times 200$.The computational results of $3 \mathrm{HL}$ patterns are compared with the GGL patterns respectively from Vassiliadis [9] and Ge [10].

\section{TABLE II. AREA OF USABLE LEFTOVERS.}

\begin{tabular}{|c|c|c|c|}
\hline ID & $L A_{3 H L}$ & $L A_{V a}$ & $L A_{G e}$ \\
\hline 1 & $\mathbf{2 8 2 0 0 0}$ & 21000 & - \\
\hline 2 & $\mathbf{8 0 0 0}$ & 0 & 4200 \\
\hline
\end{tabular}

All patterns use one plate in two instances. Table 2 shows the area of usable leftovers, where $L A_{V a}$ denotes the usable leftover area of Vassiliadis, $L A_{G e}$ denotes that of $\mathrm{Ge}$. The solution with $3 \mathrm{HL}$ patterns yields the largest usable leftovers. The average computation time for 3HL pattern is $0.068 \mathrm{~s}$, faster than that of the Vassiliadis which is 3 to 5 minutes. The computation time of Ge is not shown.

\section{V.CONCLUSION}

The paper solves the 3-staged cutting stock problem with usable leftover using a heuristic algorithm that combines the recursive technology with beam search. The computational results show that the approach is effective in seeking the optimal cutting plan with 3HL patterns in high quality. Future research may consider more objectives and allow the use of usable leftovers in a production cycle.

\section{ACKNOWLEDGEMENTS}

This research is part of Projects 61363026 and 71371058 supported by National Natural Science Foundation of China. It is also part of Project XGL090030 supported by GuangXi University.

\section{REFERENCES}

[1] Brown, A.R., Optimum packing and depletion: The computer in space and resource usage problem, New York: Macdonald - London and American Elsevier Inc, pp. 92-116, 1971.

[2] Cherri, A.C., Arenales, M.N. \&Yanasse, H.H., The one-dimensional cutting stock problem with usable leftovers - A survey. European Journal of Operational Research, 236(2), pp. 395-402, 2013.

[3] Andrade, R., Birgin, E.G., Morabito, R. \& Ronconi, D.P., MIP models for two-dimensional non-guillotine cutting problems with usable leftovers. Journal of the Operational Research Society, doi:10.1057/ jors.2013.108, 2013.

[4] Andrade, R., Birgin, E.G. \& Morabito, R., Two-stage two-dimensional guillotine cutting stock problems with usable leftover. International Transactions in Operational Research, 00, pp. 1-25, 2014.

[5] Cui, Y., Heuristic and exact algorithms for generating homogenous constrained three-staged patterns. Computers \& Operations Research, 35, pp.212-225, 2008.

[6] Hifi, M., M'Hallah, R., \& Saadi, T., Algorithms for the constrained two-staged two-dimensional cutting problem. Informs Journal on Computing, 20, pp.212-221, 2008.

[7] Hifi, M., Negre, S., Ouafi, R. \& Saadi, T., A parallel algorithm for constrained two-staged two-dimensional cutting problems. Computers \& Industrial Engineering, 62, pp.177-189, 2012.

[8] Akeb, H., Hifi, M. \& M'Hallah, R., A beam search algorithm for the circular packing problem. Computers \& Operations Research, 36, pp. 1513 -1528, 2009.

[9] Vassiliadism, V.S., Two-dimensional stock cutting and rectangle packing: 
binary tree model representation for local search optimization methods. Journal of Food Engineering, 70, pp.257-268, 2005.

[10] GE, P., QIU, Y.Q, LIU, Z.S. \& REN, P.Y., Optimized binary tree packing of guillotine problem. Computer Integrated Manufacturing Systems, 17 (2), pp. 329-337, 2011. 